\title{
Laparoscopic Right Hemicolectomy for Appendiceal Mucocele
}

\author{
Eva Kudelová ${ }^{1}$, Martin Grajciar ${ }^{1, *}$, Marek Smolar ${ }^{1}$, Michal Kalman², Ludovit Laca ${ }^{1}$
}

\begin{abstract}
Appendiceal mucocele is a rare disease with an incidence of $0.07-0.63 \%$ of all appendectomies and was first described in 1842 by Carl von Rokitansky. It is defined as an abnormal intraluminal accumulation of mucin. The clinical picture of AM can vary from asymptomatic mass in the right lower quadrant to symptoms of acute appendicitis. In some cases, AM can be found accidentally on CT performed due to other reasons or during surgery. Diagnosis consists mainly of imaging methods such as ultrasound, CT, and MRI with the finding of encapsulated cystic mass with calcifications. The main goal of surgical treatment is to remove an intact mucocele and prevent spillage of mucin into the peritoneal cavity. We present a case of large mucocele treated with laparoscopic right hemicolectomy.
\end{abstract}

\section{KEYWORDS}

mucocele; laparoscopy; appendix; hemicolectomy

AUTHOR AFFILIATIONS

${ }^{1}$ Clinic of Surgery and Transplant Centre, Jessenius Faculty of Medicine, Martin, Comenius University Bratislava, Slovakia

2 Department of Pathological Anatomy, Jessenius Faculty of Medicine, Martin, Comenius University Bratislava, Slovakia

* Corresponding author: Clinic of Surgery and Transplant Centre, Jessenius Faculty of Medicine, Comenius University in Bratislava, Kollárova 2, 03601, Martin, Slovak Republic; e-mail: martin.grajciar@unm.sk

Received: 20 February 2021

Accepted: 11 June 2021

Published online: 11 November 2021

Acta Medica (Hradec Králové) 2021; 64(3): 165-169

https://doi.org/10.14712/18059694.2021.28

(C) 2021 The Authors. This is an open-access article distributed under the terms of the Creative Commons Attribution License (http://creativecommons.org/licenses/by/4.0), which permits unrestricted use, distribution, and reproduction in any medium, provided the original author and source are credited. 


\section{INTRODUCTION}

Appendiceal mucocele is a rare disease with an incidence of $0.07-0.63 \%$ of all appendectomies (1) and was first described in 1842 by Carl von Rokitansky (2). It is defined as an abnormal intraluminal accumulation of mucin (3). A mucocele is a result of the malignant transformation of goblet cells inside the lumen of the appendix (2). Previously, appendiceal mucocele (AM) was classified into four categories (simple/retention cyst, mucosal hyperplasia, mucinous cystadenoma, and mucinous cystadenocarcinoma) (4). A consensus for classification was reached in 2016 and it is suggested that AM should be used only as a clinical term, whereas diagnosis is based on histology (1).

The clinical picture of AM can vary from asymptomatic mass in the right lower quadrant to symptoms of acute appendicitis. Patients may also present with invagination, torsion, bleeding or AM can be misdiagnosed as an adnexal mass (5) or rarely as a chronic tubo-ovarian abscess (6). In some cases, AM can be found accidentally on CT performed due to other reasons or during surgery (5). Herein, we present a case report of appendiceal mucocele in a postmenopausal female patient.

\section{CASE PRESENTATION}

A 58-year-old female patient with a positive faecal occult blood test underwent colonoscopy with a finding of round shaped lesion with a diameter of $3 \mathrm{~cm}$ protruding into the caecal lumen. The mucosa of the caecum was without any pathological signs, although a compression was presented on the caecal wall due to an extraluminally localised lesion. Subsequently, a CT scan was performed, which showed a pathological fluid collection localised in the right lower quadrant. The lesion had features of a chronic abscess with calcifications in the wall. Other visceral organs were without any specific findings. The patient underwent magnetic resonance which revealed a cystic lesion in close contact with the caecal wall with the greatest diameter of $9 \mathrm{~cm}$ with internal septae.

In this patient, a laparoscopic revision was indicated and during the procedure, a whitish elastic well-bordered tumour with the size of $9.5 \times 4 \times 4 \mathrm{~cm}$ arising from appendix basis was found. There were no metastasis or ascites present in the peritoneal cavity. The tumour was fixed to the right colon, to the right lateral abdominal wall and re-

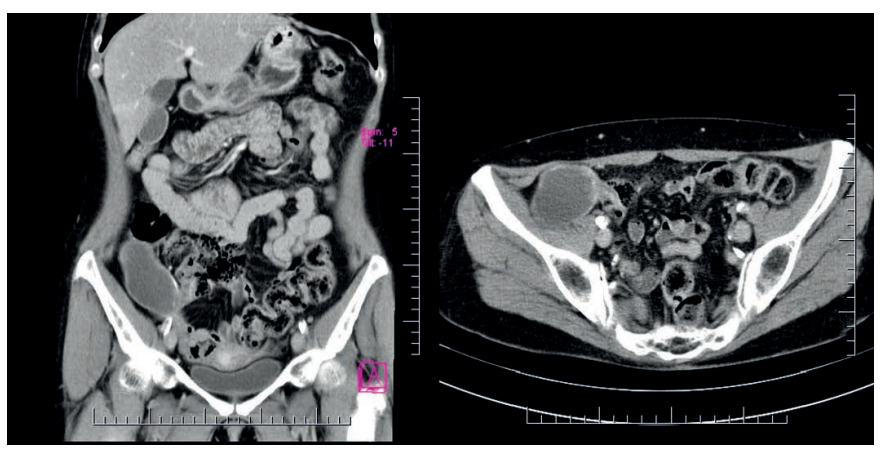

Fig. 1 Coronal and axial CT-scan showing cystic lesion with calcifications and in close contact with the caecal wall. troperitoneum. The right hemicolectomy was performed due to the tumor fixation to the adjacent structures to avoid mucocele perforation. The surgery was performed by a laparoscopic approach using the no-touch technique with the ileo-transverso-anastomosis. The specimen was removed within the endo-bag through mini-laparotomy.

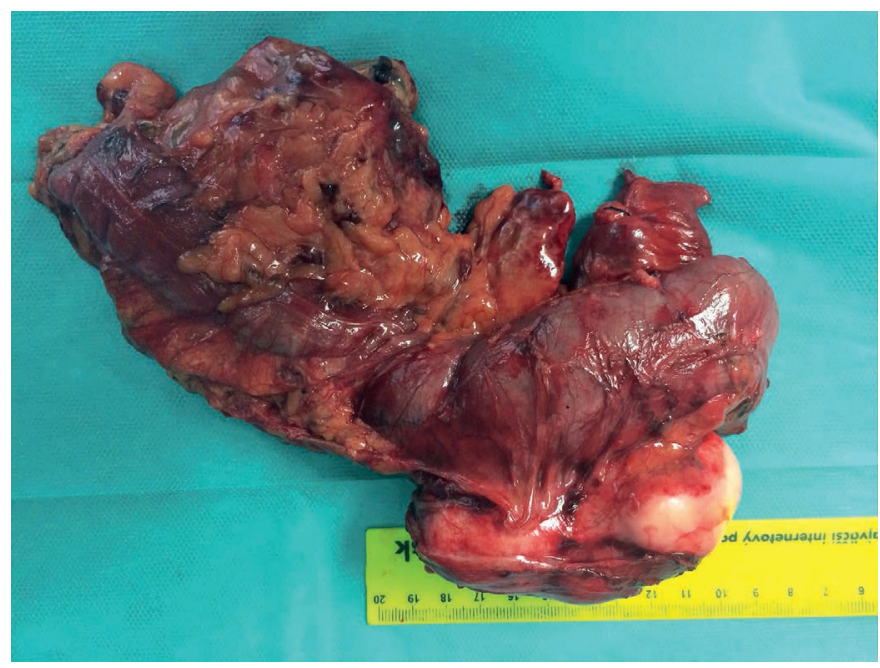

Fig. 2 Resected part of the right colon with appendiceal mucocele.

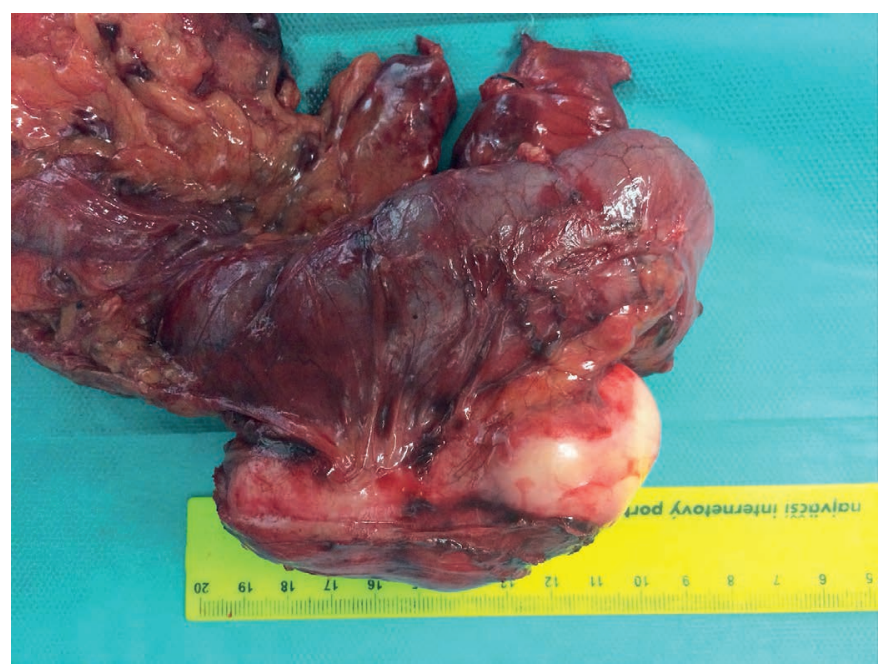

Fig. 3 Detail of appendiceal mucocele.

Histological examination confirmed low-grade mucinous neoplasm of the appendix (LAMN). The specimen was delivered to the pathology department en-block without perforation. The appendix was dilated and filled with gelatinoid material. The cystic tumour of the appendix was lined with flat and a few isolated cylindrical mucinous epithelia with focal low-grade dysplasia. The growth of the lesion had an expanding pattern with the loss of muscularis mucosae and fibrosis of submucous tissue and muscular layer without any signs of perforation or propagation of the tumour cells into the serous layer. Six lymph nodes were identified only with features of chronic antigen stimulation. Resection margins were without any neoplastic changes. Adjuvant chemotherapy was not indicated, and the patient was followed up during the first year after the surgery every 3 months and during the second year every 
6 months and underwent regular imaging and laboratory examinations. The last follow up was in January 2021 with no signs of recurrence.

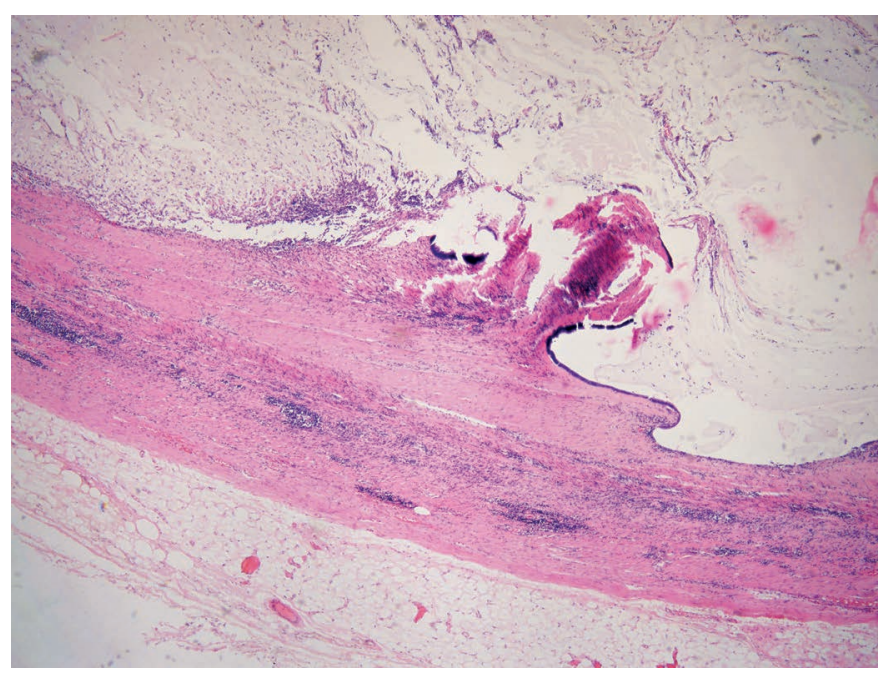

Fig. 4 Fibrosclerotic altered wall of the appendix with expansive tumour growth formed by a single-layer cylindrical epithelium with extracellular mucus formation. Most of the epithelium was denuded.

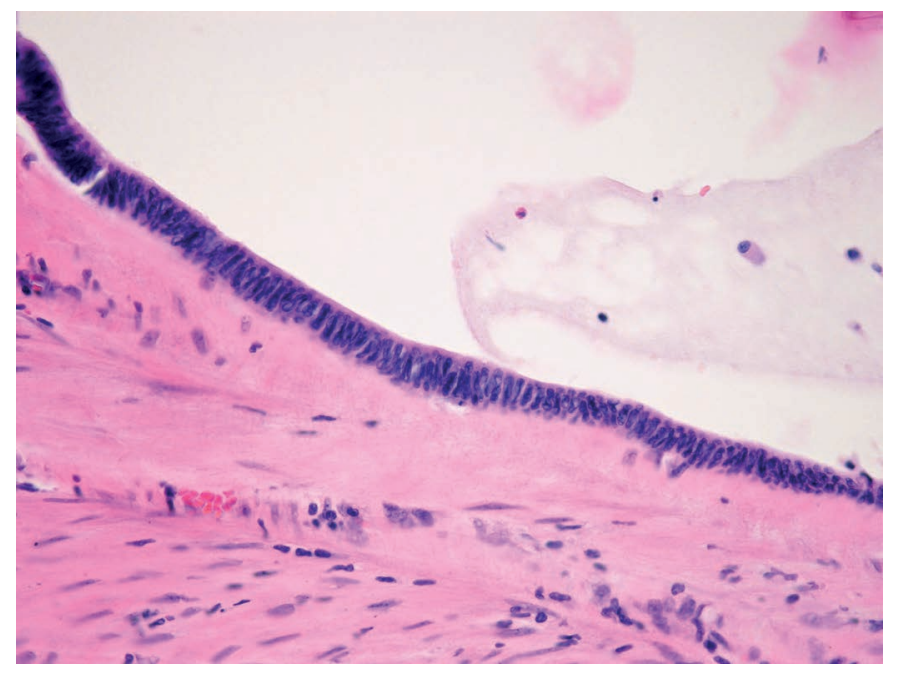

Fig. 5 Detail of a tumour lining formed by cylindrical cells with cigar-shaped hyperchromic nuclei corresponding to "low-grade" dysplasia.

\section{DISCUSSION}

Appendiceal mucocele represents an abnormal dilatation of the appendix with intraluminal mucin accumulation with an incidence of less than $1 \%$ (5). Mucocele is often diagnosed in patients over 50 years of age (4), whereas women are more often affected by this disease (7). Symptoms of AM are usually non-specific. Patients may complain of acute or chronic pain in the right lower abdominal quadrant and in some cases, a palpable mass can be present. Other common symptoms include also weight loss and changes in bowel habits (8). Some patients are admitted to the hospital due to symptoms of acute appendicitis and the diagnosis of AM is either established during surgery
(6) or it can be found accidentally on imaging studies. Our patient did not have any specific symptoms except the positive faecal occult blood test.

Mucinous neoplasm represents a broad spectrum of tumours ranging from adenoma to mucinous adenocarcinoma. According to the recent classification from 2016, non-carcinoid epithelial tumours of the appendix are categorized into eight histomorphological groups which are summarized in Table 1 (9). Modified Delphi Consensus Protocol reviewed by Carr et al. states that lesions beyond the mucosa without infiltrative invasion are classified as LAMN or HAMN. High-grade appendiceal mucinous neoplasm (HAMN) has similar features as LAMN except the high-grade cytological atypia which is characteristic for HAMN. The infiltrative invasion characterized by discohesive cells, tumor budding and desmoplastic reaction are typical for the appendiceal adenocarcinoma. The presence of desmoplasia is referred as a diagnostic criterion for distinquishing adenocarcinoma from LAMN/HAMN (9).

Tab. 1 Modified Delphi Consensus Protocol 2016 reviewed by Carr et al. Abbreviation CRC, colorectal cancer $(7,9)$.

\begin{tabular}{|l|l|}
\hline Terminology & Lesion \\
\hline $\begin{array}{l}\text { Tubular, tubovillous, villous } \\
\text { adenoma }\end{array}$ & Adenoma (traditional CRC type) \\
\hline Serrated polyp & Tumour with serrated features \\
\hline $\begin{array}{l}\text { Low-grade appendiceal } \\
\text { mucinous neoplasm }\end{array}$ & $\begin{array}{l}\text { Low-grade cytologic atypia and } \\
\text { loss of the muscularis mucosa } \\
\text { layer, pushing invasion, acellular } \\
\text { mucin in the wall, mucin outside } \\
\text { the appendix, submucosal fibrosis }\end{array}$ \\
\hline $\begin{array}{l}\text { High-grade appendiceal } \\
\text { mucinous neoplasm }\end{array}$ & $\begin{array}{l}\text { High-grade cytologic atypia } \\
\text { without infiltrative invasion }\end{array}$ \\
\hline Mucinous adenocarcinoma & $\begin{array}{l}\text { Infiltrative invasion (single cells), } \\
\text { desmoplasia }\end{array}$ \\
\hline $\begin{array}{l}\text { Mucinous adenocarcinoma } \\
\text { with signet cells }\end{array}$ & Signet cells $\leq 50 \%$ \\
\hline Signet cell carcinoma & Signet cells $\geq 50 \%$ \\
\hline Adenocarcinoma & Adenocarcinoma (non-mucinous) \\
\hline
\end{tabular}

Appendiceal mucocele in some cases represents a diagnostic challenge. Abdominal ultrasound may reveal encapsulated cystic mass with or without acoustic shadowing caused by mural calcification. A pathognomonic for AM is the "onion skin sign" which refers to echogenic layers of mucin and acoustic shadowing caused by mural calcifications inside the lumen of a mucocele $(2,10)$. Furthermore, an appendix diameter of more than $1.5 \mathrm{~cm}$ and a wall thickness of more than $6 \mathrm{~mm}$ are considered to be the threshold values for AM diagnosis (10). Dilated low-attenuated encapsulated cystic lesion of the appendix is seen on CT. Intraluminal mucin accumulation causes chronic inflammatory changes which result in wall calcification which are characteristic for AM (2). In case of secondary intra-abdominal infection, there are signs such as peri-appendiceal fat stranding, free intraperitoneal fluid, calcification, and intraluminal air-fluid level shown on CT. Features such as wall irregularity and soft-tissue thickening highly support the malignant etiol- 
ogy of AM. Magnetic resonance shows AM also as a cystic mass with various T1W1 intensity and hyperintense on T2W1 (10). Furthermore, mucocele of the appendix can be associated with synchronous colorectal neoplasms in $19-25 \%$ of cases (11). Therefore a colonoscopy should be performed before surgery to assess the extent of surgical resection $(10,12)$. A characteristic feature for AM can be found on colonoscopy known as "volcano sign" which is caused by fluctuation of protruded appendiceal ostium according to the respiratory movements (10). Tumour markers including CEA, CA 19-9 and CA-125 can be used in postoperative follow-up and their elevated levels may indicate recurrence (13), however, their diagnostic significance is low (2).

The goal of surgical treatment is to remove an intact mucocele, prevent spillage of mucin into the peritoneal cavity, and achieve negative resections margins (1). In case of spontaneous or iatrogenic perforation of $A M$, there is a high risk of pseudomyxoma peritonei development which is a severe complication with less than $20 \%$ of 5 -years survival (14). Pseudomyxoma peritonei is a clinical syndrome characterized by the presence of mucin and neoplastic epithelial cells on the parietal and visceral peritoneum. This disease is a consequence of intraperitoneal dissemination from mucin-producing tumours (7). The treatment of PMP consists of cytoreductive surgery followed by hyperthermic intraperitoneal chemotherapy (15).

There are still no clear guidelines for surgical treatment of mucocele, however, Dhage-Ivatury et al. (16) and Kim et al. (17) created a scheme for the selection of the type of surgery. Several factors, which should be considered before the surgery, are summarized in table 1 (5). Frozen section examination of resection margins and sentinel lymph node (SLN) from mesoappendix is often useful and necessary to assess the extent of surgery. Right hemicolectomy is not indicated if SLN is without metastasis (18). Historically, laparotomy has been considered as the preferred surgical approach for mucocele treatment (4), however, there are several reports in the literature of successful laparoscopic mucocele resections. General principles of AM removal are the same regardless of the surgical approach. The operation should be performed carefully with an emphasis on the no-touch technique. An endo-bag must be used to prevent rupture of AM and port-site metastasis, while the surgeon should be experienced enough with laparoscopy (19). The prognosis of AM depends on several factors such as symptoms, histological parameters, perforation, increased tumour markers levels, and positive resection margins (20).

\section{CONCLUSION}

Appendiceal mucocele is a rare disease that can be asymptomatic or resembles acute appendicitis. Pre-operative diagnosis consists of imaging methods such as ultrasound, CT, MRI, or colonoscopy is used. The main goal of surgical treatment is to remove an intact mucocele and prevent spillage of mucin into the peritoneal cavity.

\section{REFERENCES}

1. Cestino L, Festa F, Cavuoti G, et al. Appendiceal mucocele: three cases with different clinical presentation and review of literature. J Surg Case Rep 2020; 2020(9): rjaa344.

2. Kwak HD, Ju JK. A prospective study of discrepancy between clinical and pathological diagnosis of appendiceal mucinous neoplasm. Ann Surg Treat Res 2020; 98(3): 124-9.

3. Sakata S, Moran BJ. What is a "mucocele" of the appendix and how are these lesions best managed? Beware the wolf in sheep's clothing. Colorectal Dis Off J Assoc Coloproctology G B Irel 2019; 21(11): 1237-9.

4. Şentürk M, Yavuz Y, Alkan S, Kafadar MT. The Investigation of 14 Appendiceal Mucocele Cases Encountered in 4850 Appendectomy Patients. J Gastrointest Cancer 2021; 52(2): 701-5.

5. Sun P, Jiang F, Sun H, et al. Minimally invasive surgery for appendiceal intussusception caused by mucocele of the appendix: case report and review of the literature. J Gastrointest Oncol 2020; 11(1): $102-7$.

6. Cubro H, Cengic V, Burina N, Kravic Z, Beciragic E, Vranic S. Mucocele of the appendix presenting as an exacerbated chronic tubo-ovarian abscess: A case report and comprehensive review of the literature. Medicine (Baltimore) 2019; 98(39): e17149.

7. Gündoğar Ö, Kımıloğlu E, Komut N, et al. Evaluation of appendiceal mucinous neoplasms with a new classification system and literature review. Turk J Gastroenterol Off J Turk Soc Gastroenterol 2018; 29(5): 533-42.

8. Arnason T, Kamionek M, Yang M, Yantiss RK, Misdraji J. Significance of proximal margin involvement in low-grade appendiceal mucinous neoplasms. Arch Pathol Lab Med 2015; 139(4): 518-21.

9. Carr NJ, Cecil TD, Mohamed F, et al. A Consensus for Classification and Pathologic Reporting of Pseudomyxoma Peritonei and Associated Appendiceal Neoplasia: The Results of the Peritoneal Surface Oncology Group International (PSOGI) Modified Delphi Process. Am J Surg Pathol 2016; 40(1): 14-26.

10. Sharma P, Soin P, Chugh M, Goyal P. Dilated Appendix: Is There More to It? Case Report and Brief Review of Literature with Radiologic-Pathological Correlation. J Clin Imaging Sci 2019; 9: 9.

11. Alghamdi AO, Aldossary MY, Alsawidan M, AlBahar S. Low grade appendiceal mucinous neoplasm mimicking an ovarian cyst: A case report. Int J Surg Case Rep 2020; 70: 145-8.

Tab. 2 Factors determining the extent of surgery. Abbreviations AM, appendiceal mucocele; LN, lymph node $(5,16,17)$.

\begin{tabular}{|l|l|l|l|}
\hline \multicolumn{2}{|l}{ Appendectomy } & Partial cecectomy & Right hemicolectomy \\
\hline \multirow{4}{*}{ Factors } & Non-perforated & Non-perforated & Non-perforated \\
\cline { 2 - 4 } & Intact appendiceal base & Broad, protruding AM into the caecal wall & AM invades the caecal wall or ileum \\
\cline { 2 - 4 } & Negative cytology & Negative/positive cytology & An adequate resection margin cannot be obtained \\
\cline { 2 - 4 } & Negative lymph nodes & Negative/positive margins of appendiceal stump & Positive cytology \\
\cline { 2 - 4 } & & Negative appendiceal LN & Positive resection margin \\
\cline { 2 - 4 } & & & Positive margin of appendiceal stump \\
\cline { 2 - 4 } & & & Positive appendiceal LN \\
\hline
\end{tabular}


12. Foula MS, Alardhi AM, Othman SA, Mirza Gari MK. Laparoscopic management of appendicular mucinous cystadenoma, case report. Int J Surg Case Rep 2019; 54: 87-9.

13. Padmanaban V, Morano WF, Gleeson E, et al. Incidentally discovered low-grade appendiceal mucinous neoplasm: a precursor to pseudomyxoma peritonei. Clin Case Rep 2016; 4(12): 1112-6.

14. Pantiora EV, Massaras D, Koutalas J, Bagiasta A, Kontis EA, Fragulidis GP. Low-grade Appendiceal Mucinous Neoplasm Presenting as Adnexal Mass: A Case Report. Cureus 2018; 10(11): e3568.

15. Sullivan BJ, Bolton N, Sarpel U, Magge D. A unique presentation of superinfected pseudomyxoma peritonei secondary to a low-grade appendiceal mucinous neoplasm. World J Surg Oncol 2019; 17(1): 34 .

16. Dhage-Ivatury S, Sugarbaker $\mathrm{PH}$. Update on the surgical approach to mucocele of the appendix. J Am Coll Surg 2006; 202 (4): 680-4.
17. Kim TK, Park JH, Kim JY, et al. Safety and feasibility of laparoscopic surgery for appendiceal mucocele: a multicenter study. Surg Endosc 2018; $32(11): 4408-14$

18. González-Moreno S, Sugarbaker PH. Radical appendectomy as an alternative to right colon resection in patients with epithelial appendiceal neoplasms. Surg Oncol 2017; 26(1): 86-90.

19. Park B-S, Shin DH, Kim D-I, Son GM, Kim HS. Appendiceal intussusception requiring an ileocecectomy: a case report and comment on the optimal surgery. BMC Surg 2018; 18(1): 48.

20. Shin R, Chai YJ, Park JW, et al. Ultimate Clinical Outcomes of Appendiceal Mucinous Neoplasm of Uncertain Malignant Potential. Ann Surg Oncol 2017; 24(4): 974-82. 